REVISIONES

Rev Obstet Ginecol Venez. 2021; 81 (2): 155-161

https://doi.org/10.51288/00810209

\title{
Rol de la citorreducción secundaria en cáncer de ovario recurrente
}

\author{
Ernesto Lara, ${ }^{1}$ (D) Franco Calderaro Di Ruggiero. ${ }^{2}$
}

\begin{abstract}
RESUMEN
El cáncer de ovario constituye una de las enfermedades más desafiantes en ginecología oncológica. La mayoría de las pacientes son diagnosticadas en estadios avanzados y se desarrolla enfermedad recurrente con elevada frecuencia. Se han publicado pocos estudios prospectivos centrados en los efectos de la cirugía en el cáncer de ovario recurrente, los datos disponibles muestran mejoras en el pronóstico debido a la citorreducción completa en el contexto de la recurrencia, para ello, la selección de pacientes es fundamental. Aunque la cirugía es crucial en la terapia de pacientes con cáncer de ovario primario y la masa tumoral residual posoperatoria es el factor pronóstico más relevante, la eficacia de la citorreducción secundaria ha estado sujeta a controversias. La presente revisión tiene como objetivo precisar el rol de la citorreducción secundaria en el cáncer de ovario recurrente, basados en la evidencia publicada para, de esta manera, garantizar la terapéutica más adecuada.
\end{abstract}

Palabras clave: Cáncer de ovario, Citorreducción primaria, Citorreduccion secundaria, Cáncer de ovario recurrente.

\section{SUMMARY}

Ovarian cancer is one of the most challenging diseases in gynecologic oncology. Most patients are diagnosed in advanced stages and recurrent disease develops with high frequency. Few prospective studies have been published focusing on the effects of surgery on recurrent ovarian cancer, the available data show improvements in prognosis due to complete cytoreduction in the context of recurrence, for this, the selection of patients is essential. Although surgery is crucial in the therapy of patients with primary ovarian cancer and postoperative residual tumor mass is the most relevant prognostic factor, the efficacy of secondary cytoreduction has been subject to controversy. The present review aims to clarify the role of secondary cytoreduction in recurrent ovarian cancer, based on the published evidence to ensure the most appropriate therapy.

Key words: Ovarian cancer, Primary cytoreduction, Secondary cytoreduction, Recurrent ovarian cancer

\section{INTRODUCCIÓN}

El cáncer de ovario (CO) es la octava causa más común de muerte por cáncer en las mujeres y la segunda causa de mortalidad por cáncer ginecológico a nivel mundial (1). En los Estados Unidos, causa más muertes que

${ }^{1}$ Ginecólogo Oncólogo, Ginecólogo Obstetra. Caracas, Venezuela. ${ }^{2}$ Doctor en Ciencias Médicas. Cirujano Oncólogo, Ginecólogo Oncólogo, Jefe del servicio de Ginecología Oncológica, Servicio Oncológico Hospitalario IVSS. Caracas, Venezuela. cualquier otro cáncer del tracto genital femenino, con más de 21750 casos nuevos y 13940 muertes estimadas en 2020 (2). En Venezuela, se ubica como la segunda causa de defunción por cáncer originado en el tracto genital femenino (3).

A pesar de la evolución de las diferentes modalidades de tratamiento, el CO constituye una de las enfermedades más desafiantes en ginecología oncológica, la mayoría de las pacientes son diagnosticadas en estadios avanzados $\mathrm{y}$, desafortunadamente, la enfermedad recurrente se desarrolla en más del $80 \%$ de las mujeres y las tasas a 10 años de supervivencia libre de 
enfermedad entre pacientes con enfermedad recurrente están por debajo del $15 \%(4,5)$. Aproximadamente el $22 \%$ de las recaídas se presentan en 6 meses, mientras que la mayoría de las pacientes experimentan la llamada recaída sensible al platino, con un intervalo de más de 6 meses después de la quimioterapia basada en platino (6). La cirugía y la quimioterapia basada en platino son los pilares del tratamiento multimodal, en el contexto de la enfermedad primaria, y la masa tumoral residual posoperatoria es el factor pronóstico más relevante $(7,8)$. Aunque la citorreducción primaria o la cirugía de intervalo han sido bien establecidas con un rol esencial, la eficacia de la citorreduccion secundaria (CS) ha estado sujeta a controversias.

Teóricamente, el esfuerzo quirúrgico máximo puede ayudar a superar la resistencia farmacológica intrínseca, aumentar la perfusión del fármaco, mejorar la respuesta inmunológica del huésped, aumentar la fracción de crecimiento de las células tumorales y evitar la resistencia farmacológica adquirida después de la terapia sistémica adyuvante basada en platino y taxano (9 - 11). La CS aparece a principios de la década de 1990 como una opción de tratamiento (12) y numerosas revisiones retrospectivas de una y de varias instituciones, así como metaanálisis, han reforzado el apoyo al procedimiento, mostrando que las pacientes que tuvieron el mayor beneficio fueron aquellas con poca o ninguna enfermedad residual posoperatoria y aquellas consideradas sensibles al platino (13-17).

Adicionalmente, la evidencia prospectiva es controvertida, basada en los resultados de dos ensayos aleatorizados fase 3 , de reciente publicación, como son el GOG 213 (18) y el DESKTOP III (19), por lo cual, el motivo de la presente revisión, es precisar el rol de la CS en el cáncer de ovario recurrente (COR), basados en la evidencia publicada.

\section{Visión general}

La CS, se define como un procedimiento que se realiza para extirpar la mayor cantidad de masa tumoral posible y prolongar la supervivencia en pacientes con enfermedad recurrente después de completar el tratamiento primario, cirugía con o sin quimioterapia, y de un periodo de, al menos, 6 meses sin evidencia de enfermedad (20).

El primer informe de CS en COR, fue realizado en 1983 por Berek y cols. (21). En este análisis retrospectivo, los autores encontraron que la supervivencia media era de 20 meses en pacientes con citorreducción óptima (definida como $<1,5 \mathrm{~cm}$ ), frente a 5 meses en pacientes con reducción de volumen subóptima.

En 2001, Scarabelli y cols. (22), intentaron identificar los factores pronósticos para la supervivencia en el COR mediante la realización de un estudio prospectivo con 149 pacientes, que cumplieron con los criterios de selección para CS, determinando que la CS completa fue el principal factor predictivo.

Igualmente, diferentes estudios han sugerido que el beneficio de la cirugía para el COR podría limitarse a pacientes, en quienes se puede lograr una citorreducción completa. La mayoría de las series reportan la resección completa como un factor pronóstico independiente para mejorar la supervivencia después de la cirugía (12, 22-30), resaltando así la importancia de un proceso de selección adecuado para considerar la CS en el manejo de estas pacientes.

\section{Predicción de citorreducción completa}

La CS parece beneficiar a pacientes bien seleccionadas cuando se logra citorredución completa (R0). Por lo tanto, muchos esfuerzos se han centrado en cómo identificar a las pacientes en quienes es probable que la CS conduzca a una resección completa. En 1998, la segunda conferencia de consenso sobre el cáncer de ovario describió los siguientes factores para la identificación adecuada de pacientes para cirugía con 
enfermedad recurrente: periodo libre de progresión mayor a 12 meses, respuesta a la primera línea de quimioterapia, que la resección completa parezca factible, buen estatus performance y edad temprana (31).

Asimismo, en 2006, Chi y cols. (17), en el Memorial Sloan Kettering (MSK), reportaron una tasa de resección completa del $41 \%$ en un estudio retrospectivo de 153 pacientes sometidas a CS. Basado en su análisis, la CS fue recomendada en pacientes con un solo sitio de recurrencia, independientemente del periodo libre de enfermedad; mujeres con múltiples sitios de recurrencia, pero sin carcinomatosis y periodo libre de enfermedad (PLE) mayor a 12 meses y mujeres con carcinomatosis pero con PLE mayor a 30 meses.

El Arbeidsgemeinschaft Gynaekologische Onkologie Ovarian Committee (AGO OC), realizó en 2005 un estudio multicéntrico titulado Descriptive Evaluation of preoperative selection kriteria for operability in recurrent ovarian cancer (DESKTOP OVAR), basado en datos retrospectivos de 267 pacientes sometidas a CS. Identificaron un panel de criterios preoperatorios que predecían significativamente la citorreducción completa; estos fueron: buen estado funcional - Eastern Cooperative Oncology Group (ECOG) 0, resección completa en la primera cirugía y ausencia de ascitis. Las pacientes con estos 3 criterios se consideran AGO score positivo. Basado en la combinación de estos criterios de selección preoperatoria, se logró predecir citorreducción completa en el $79 \%$ de los casos (23). Posteriormente se realizó el DESKTOP II (32) con el objetivo de validar prospectivamente los resultados de DESKTOP I, con la incorporación de estos criterios, encontrando que un AGO score positivo, podría predecir citorreducción completa en el $76 \%$ de las pacientes.

Por otro lado, Tian y cols. (20) realizaron una revisión retrospectiva internacional de pacientes de 9 cohortes diferentes, que abarcan desde 1982 hasta 2006, desarrollando un modelo de riesgo con 6 factores para predecir la citorreducción completa: estadio FIGO (I/II o III/IV), enfermedad residual después de la citorreducción primaria $(0 \mathrm{o}>0)$, supervivencia libre de progresión (<16 o > 16 meses), ECOG ( 0-1 o 2-3), CA-125 ( $\leq 105 \mathrm{o}>105 \mathrm{U} / \mathrm{mL}$ ) y ascitis (ausente o presente). Estas variables se introdujeron en el modelo de riesgo y se les asignaron puntuaciones que iban de 0 a 11,9. Las pacientes con una puntuación total de 4,7 o menos se clasificaron en el grupo de bajo riesgo. La citorreducción completa se logró en el $53 \%$ de las pacientes de bajo riesgo frente a solo el $20,1 \%$ de las pacientes de alto riesgo. Los investigadores también realizaron, una pequeña validación externa que demostró una sensibilidad del $83 \%$ y una especificidad del 57,6\%. Con base a la evidencia de su cohorte, se debe recomendar la CS a las pacientes que tienen un riesgo bajo.

Dentro de todos estos sistemas de puntuación para la predicción de citorreducción R0, el AGO Score continúa siendo el único conjunto de criterios de selección validado prospectivamente.

\section{Eficacia de la CS, evidencia retrospectiva y prospectiva}

Desde 2003, se han realizado varios estudios retrospectivos que compararon directamente la CS con quimioterapia versus quimioterapia sola. Los resultados de estos estudios respaldaron unánimemente el hallazgo de que la supervivencia mejoró con la adición de CS al régimen de tratamiento (33 - 43). Aunque algunos de los estudios han intentado comparar grupos similares de pacientes, se ven debilitados por la falta de aleatorización, lo que lleva a un sesgo de selección.

Desde 2005, al menos 5 estudios prospectivos aleatorizados que comparan $\mathrm{CS}$ con quimioterapia han comenzado a realizarse $(18,19,44)$. Dos de estos 
ensayos se cerraron tempranamente debido a la poca cantidad de pacientes reclutadas. Solo GOG-0213 (18) y DESKTOP III (19), han publicado sus resultados finales y el SOC 1 (44) se encuentra en curso.

GOG-0213 (18) es un estudio aleatorizado, internacional, multicéntrico, fase 3 , que evaluó la CS en COR sensible al platino. Se asignaron, al azar, pacientes con COR que habían recibido una terapia previa, tenían un intervalo durante el cual no se utilizó quimioterapia basada en platino (intervalo sin platino) de 6 meses o más y tenían enfermedad resecable, determinada por el investigador, para someterse a CS más quimioterapia con base en platino o solo recibir quimioterapia. La quimioterapia adyuvante (paclitaxel-carboplatino o gemcitabina-carboplatino) y el uso de bevacizumab quedaron a criterio del investigador. El primer objetivo era la supervivencia global (SG). Se incluyeron 485 pacientes, 240 a CS antes de la quimioterapia y 245 a quimioterapia sola. La mediana de seguimiento fue de 48,1 meses. La CS se logró en el $67 \%$ de las pacientes asignadas a cirugía y que se sometieron al procedimiento. La SG fue de 50,6 meses para las que se sometieron a CS en comparación con 64,7 meses para los que se sometieron solo a quimioterapia. La supervivencia libre de progresión fue similar, 18,9 meses para CS y 16,2 meses para el grupo que recibió solo quimioterapia. Concluyeron que la CS seguida de quimioterapia, no resultó en una supervivencia global más prolongada con respecto a la quimioterapia sola.

DESKTOP III (19) evaluó el papel de la CS en el cáncer de ovario recurrente, los resultados finales fueron presentados en la reunión de la American Society of Clinical Oncology (ASCO) 2020. Las pacientes con COR y primera recaída después de más de 6 meses de intervalo sin platino, eran elegibles si presentaban una puntuación AGO positiva (ECOG 0, ascitis $\leq$ $500 \mathrm{ml}$ y resección completa en la cirugía inicial), y fueron aleatorizados a quimioterapia de segunda línea sola versus cirugía citorreductora seguida de la misma quimioterapia. Se incluyeron 407 pacientes, 206 fueron asignados al brazo de cirugía, de las cuales finalmente 187 (91 \%) fueron operadas, lográndose una citorreducción completa en el $75 \%$. La SG fue de 53,7 meses para el grupo sometida a cirugía y 46,2 meses para el grupo que no recibió cirugía; el PLE fue 18,4 y 14 meses a favor del grupo sometido a cirugía. Un análisis según el tratamiento mostró un beneficio en cuanto a la SG superior a 12 meses para las pacientes con resección completa, en comparación con las pacientes sin cirugía (mediana 60,7 frente a 46,2 meses); las pacientes con cirugía y resección incompleta incluso empeoraron (mediana 28,8 meses). Este es el primer estudio que demuestra un beneficio de supervivencia significativo de la cirugía en $\mathrm{CO}$ en pacientes con: primera recaída e intervalo libre de platinos de más de 6 meses y seleccionadas por un puntaje AGO positivo. El beneficio se observó exclusivamente en pacientes con citorreducción completa, lo que indica la importancia tanto de la selección óptima de pacientes, como el manejo en centros con experiencia y una alta probabilidad de lograr una resección completa.

El ensayo SOC 1 (44) es un estudio chino, aleatorizado fase III de CS en COR. Las pacientes con primera recaída de $\mathrm{CO}$ después de más de 6 meses de intervalo libre de platino, fueron elegibles si se predecía una cirugía R0 por iMODEL score combinado con imágenes por tomografía por emisión de positrones (CT-PET) y fueron aleatorizadas a CS seguida con quimioterapia vs quimioterapia sola. Los objetivos principales a evaluar son sobrevida global y periodo libre de enfermedad. Los resultados parciales fueron presentados en el ASCO meeting 2020, encontrando que 357 pacientes fueron aleatorizadas entre 2012 -2019, (R0) se logró en el 76,7 \% y el PLE fue 17,4 m y $11,9 \mathrm{~m}$ en el grupo sometido a cirugía y no sometido a cirugía respectivamente $(\mathrm{p}<0,0001)$. Los resultados de la SG aún no están disponibles. Con base en estos resultados, la CS en pacientes seleccionadas aumenta significativamente el periodo libre de enfermedad. 


\section{CONCLUSIONES}

La CS representa un enfoque de tratamiento viable para pacientes seleccionadas, en las cuales es fundamental la aplicación de factores predictivos fiables para la resección completa de la enfermedad. La CS demostró beneficio en pacientes con COR cuando: es la primera recaída, las pacientes son platino sensibles y seleccionadas adecuadamente, con probabilidad de lograr cirugía (R0).

\section{REFERENCIAS}

1. Bray F, Ferlay J, Soerjomataram I, Siegel RL, Torre LA, Jemal A. Global cancer statistics 2018: GLOBOCAN estimates of incidence and mortality worldwide for 36 cancers in 185 countries. CA Cancer J Clin. 2018; 68 (6):394-424. doi: 10.3322/caac.21492.

2. Siegel RL, Miller KD, Jemal A. Cancer statistics, 2020. CA Cancer J Clin. 2020; 70(1):7-30. doi: 10.3322/ caac. 21590.

3. World Health Organization [Internet]. Ginebra: The Global Cancer Observatory. Venezuela, Bolivarian Republic of Source: Globocan; 2018 [consultado el 29 de julio de 2020] Disponible en: https://gco.iarc. $\mathrm{fr} /$ today/data/factsheets/populations/862-venezuelabolivarian-republic-of-fact-sheets.pdf.

4. Berek JS, Kehoe ST, Kumar L, Friedlander M. Cancer of the ovary, fallopian tube, and peritoneum. Int $\mathrm{J}$ Gynaecol Obstet. 2018; 143(Suppl2):59-78. doi: 10.1002/ijgo.12614.

5. Dood RL, Zhao Y, Armbruster SD, Coleman RL, Tworoger S, Sood AK, et al. Defining Survivorship Trajectories Across Patients With Solid Tumors: An Evidence-Based Approach. JAMA Oncol. 2018; 4(11):1519-1526. doi: 10.1001/jamaoncol.2018.2761.

6. du Bois A, Reuss A, Pujade-Lauraine E, Harter P, Ray-Coquard I, Pfisterer J. Role of surgical outcome as prognostic factor in advanced epithelial ovarian cancer: a combined exploratory analysis of 3 prospectively randomized phase 3 multicenter trials: by the Arbeitsgemeinschaft Gynaekologische Onkologie Studiengruppe Ovarialkarzinom (AGO-OVAR) and the Groupe d'Investigateurs Nationaux Pour les Etudes des Cancers de l'Ovaire (GINECO). Cancer. 2009; 115(6):1234-1244. doi: 10.1002/cncr.24149.

7. Griffiths CT. Surgical resection of tumor bulk in the primary treatment of ovarian carcinoma. Natl Cancer Inst Monogr. 1975; 42:101-104.

8. Bristow RE, Tomacruz RS, Armstrong DK, Trimble EL, Montz FJ. Survival effect of maximal cytoreductive surgery for advanced ovarian carcinoma during the platinum era: a meta-analysis. J Clin Oncol. 2002; 20(5):1248-1259. doi: 10.1200/JCO.2002.20.5.1248.

9. Skipper HE. Adjuvant chemotherapy. Cancer. 1978; 41(3):936-940. doi: $10.1002 / 1097-0142(197803) 41: 3<936:$ :aidcncr2820410322>3.0.co;2-b.

10. Goldie JH, Coldman AJ. A mathematic model for relating the drug sensitivity of tumors to their spontaneous mutation rate. Cancer Treat Rep. 1979; 63(11-12):1727-1733.

11. Norton L, Simon R. Tumor size, sensitivity to therapy, and design of treatment schedules. Cancer Treat Rep. 1977; 61(7):1307-1317.

12. Jänicke $F$, Hölscher $M$, Kuhn W, von Hugo R, Pache $\mathrm{L}$, Siewert JR, et al. Radical surgical procedure improves survival time in patients with recurrent ovarian cancer. Cancer. 1992; 70 (8):2129-2136. doi: $\quad 10.1002 / 1097-0142(19921015) 70: 8<2129:: a i d-$ cncr2820700820>3.0.co;2-u.

13. Bristow RE, Puri I, Chi DS. Cytoreductive surgery for recurrent ovarian cancer: a meta-analysis. Gynecol Oncol. 2009; 112(1):265-274. doi: 10.1016/j. ygyno.2008.08.033.

14. Bommert M, Harter P, Heitz F, du Bois A. When should Surgery be used for Recurrent Ovarian Carcinoma? Clin Oncol (R Coll Radiol). 2018; 30(8):493-497. doi: 10.1016/j.clon.2018.04.006.

15. Santillan A, Karam AK, Li AJ, Giuntoli R 2nd, Gardner GJ, Cass I, et al. Secondary cytoreductive surgery for isolated nodal recurrence in patients with epithelial ovarian cancer. Gynecol Oncol. 2007; 104(3):686-690. doi: 10.1016/j.ygyno.2006.10.020.

16. Zang RY, Harter P, Chi DS, Sehouli J, Jiang R, Tropé CG, et al. Predictors of survival in patients with recurrent ovarian cancer undergoing secondary cytoreductive surgery based on the pooled analysis of an international collaborative cohort. Br J Cancer. 2011; 105(7):890896. doi: 10.1038/bjc.2011.328.

17. Chi DS, McCaughty K, Diaz JP, Huh J, Schwabenbauer $\mathrm{S}$, Hummer AJ, et al. Guidelines and selection criteria for secondary cytoreductive surgery in patients with recurrent, platinum-sensitive epithelial ovarian carcinoma. Cancer. 2006; 106(9):1933-1939. doi: 10.1002/cncr.21845.

18. Coleman RL, Spirtos NM, Enserro D, Herzog TJ, 


\section{ROL DE LA CITORREDUCCIÓN SECUNDARIA EN CÁNCER DE OVARIO RECURRENTE}

Sabbatini P, Armstrong DK, et al. Secondary Surgical Cytoreduction for Recurrent Ovarian Cancer. N Engl J Med. 2019; 381(20):1929-1939. doi: 10.1056/ NEJMoa1902626.

19. du Bois A, Sehouli J, Vergote I, Ferron G, Reuss A, Meier $\mathrm{W}$, et al. Randomized phase III study to evaluate the impact of secondary cytoreductive surgery in recurrent ovarian cancer: Final analysis of AGO DESKTOP III/ENGOT-ov20 [Internet]. American Society of Clinical Oncology; 2020 [consultado el 21 de agosto de 2020] Disponible: https://s3.amazonaws.com/files. oncologymeetings.org/prod/s3fs-public/2020-05/ AM20-GYNECOLOGIC-CANCER.pdf?null.

20. Tian WJ, Chi DS, Sehouli J, Tropé CG, Jiang R, Ayhan A, et al. A risk model for secondary cytoreductive surgery in recurrent ovarian cancer: an evidence-based proposal for patient selection. Ann Surg Oncol. 2012; 19(2):597-604. doi: 10.1245/s10434-011-1873-2.

21. Berek JS, Hacker NF, Lagasse LD, Nieberg RK, Elashoff RM. Survival of patients following secondary cytoreductive surgery in ovarian cancer. Obstet Gynecol [Internet]. 1983 [consultado 21 de agosto de 2020]; 61(2):189-193. Disponible en: http://intl.greenjournal. org/cgi/content/abstract/61/2/189

22. Scarabelli C, Gallo A, Carbone A. Secondary cytoreductive surgery for patients with recurrent epithelial ovarian carcinoma. Gynecol Oncol. 2001; 83(3):504-512. doi: 10.1006/gyno.2001.6404.

23. Harter P, du Bois A, Hahmann M, Hasenburg A, Burges A, Loibl S, et al. Surgery in recurrent ovarian cancer: the Arbeitsgemeinschaft Gynaekologische Onkologie (AGO) DESKTOP OVAR trial. Ann Surg Oncol. 2006; 13(12):1702-1710. doi: 10.1245/s10434-006-9058-0.

24. Eisenkop SM, Friedman RL, Spirtos NM. The role of secondary cytoreductive surgery in the treatment of patients with recurrent epithelial ovarian carcinoma. Cancer. 2000; 88(1):144- 153. doi: 10.1002/(sici)10970142(20000101)88:1<144::aid-cncr20>3.3.co;2-o.

25. Gronlund B, Lundvall L, Christensen IJ, Knudsen JB, Høgdall C. Surgical cytoreduction in recurrent ovarian carcinoma in patients with complete response to paclitaxel-platinum. Eur J Surg Oncol. 2005; 31(1):6773. doi: 10.1016/j.ejso.2004.08.016.

26. Sehouli J, Richter R, Braicu EI, Bühling KJ, Bahra $\mathrm{M}$, Neuhaus $\mathrm{P}$, et al. Role of secondary cytoreductive surgery in ovarian cancer relapse: who will benefit? A systematic analysis of 240 consecutive patients. J Surg Oncol. 2010; 102(6):656-662. doi: 10.1002/jso.21652.

27. Leitao MM Jr, Kardos S, Barakat RR, Chi DS. Tertiary cytoreduction in patients with recurrent ovarian carcinoma. Gynecol Oncol. 2004; 95(1):181-188. doi: 10.1016/j.ygyno.2004.07.033.

28. Tay EH, Grant PT, Gebski V, Hacker NF. Secondary cytoreductive surgery for recurrent epithelial ovarian cancer. Obstet Gynecol. 2002; 99(6):1008-1013. doi: 10.1016/s0029-7844(02)01977-4.

29. Zang RY, Li ZT, Tang J, Cheng X, Cai SM, Zhang ZY, et al. Secondary cytoreductive surgery for patients with relapsed epithelial ovarian carcinoma: who benefits? Cancer. 2004; 100(6):1152-1161. doi: 10.1002/ cncr.20106.

30. Benedetti Panici P, De Vivo A, Bellati F, Manci N, Perniola G, Basile S, et al. Secondary cytoreductive surgery in patients with platinum-sensitive recurrent ovarian cancer. Ann Surg Oncol. 2007; 14 (3):11361142. doi: 10.1245/s10434-006-9273-8.

31. Berek JS, Bertelsen K, du Bois A, Brady MF, Carmichael J, Eisenhauer EA, et al. Advanced epithelial ovarian cancer: 1998 consensus statements. Ann Oncol. 1999; 10(Suppl 1):87-92. doi: 10.1023/a:1008323922057.

32. Harter P, Sehouli J, Reuss A, Hasenburg A, Scambia G, Cibula D, et al. Prospective validation study of a predictive score for operability of recurrent ovarian cancer: the Multicenter Intergroup Study DESKTOP II. A project of the AGO Kommission OVAR, AGO Study Group, NOGGO, AGO-Austria, and MITO. Int J Gynecol Cancer. 2011; 21(2): 289-295. doi: 10.1097/ IGC.0b013e31820aaafd.

33. Güngör M, Ortaç F, Arvas M, Kösebay D, Sönmezer M, Köse K. The role of secondary cytoreductive surgery for recurrent ovarian cancer. Gynecol Oncol. 2005; 97(1):74-79. doi: 10.1016/j.ygyno.2004.11.057.

34. Matsumoto A, Higuchi T, Yura S, Mandai M, Kariya $\mathrm{M}$, Takakura $\mathrm{K}$, et al. Role of salvage cytoreductive surgery in the treatment of patients with recurrent ovarian cancer after platinum-based chemotherapy. J Obstet Gynaecol Res. 2006; 32(6):580-587. doi: 10.1111/j.1447-0756.2006.00460.x.

35. Oksefjell H, Sandstad B, Tropé C. The role of secondary cytoreduction in the management of the first relapse in epithelial ovarian cancer. Ann Oncol. 2009; 20(2):286293. doi: 10.1093/annonc/mdn591. Epub 2008 Aug 25.

36. Gadducci A, Cosio S, Zola P, Sostegni B, Ferrero AM, Teti $\mathrm{G}$, et al. The clinical outcome of epithelial ovarian cancer patients with apparently isolated lymph node recurrence: a multicenter retrospective Italian study. Gynecol Oncol. 2010; 116(3):358-363. doi: 10.1016/j.ygyno.2009.11.008.

37. Classe JM, Jaffre I, Frenel JS, Bordes V, Dejode 
M, Dravet F, et al. Prognostic factors for patients treated for a recurrent FIGO stage III ovarian cancer: a retrospective study of 108 cases. Eur J Surg Oncol. 2011; 37(11):971-977. doi: 10.1016/j.ejso.2011.08.138.

38. Lee CK, Lord S, Grunewald T, Gebski V, HardyBessard AC, Sehouli J, et al. Impact of secondary cytoreductive surgery on survival in patients with platinum sensitive recurrent ovarian cancer: analysis of the CALYPSO trial. Gynecol Oncol. 2015; 136(1):1824. doi: 10.1016/j.ygyno.2014.09.017.

39. da Costa AA, Valadares CV, Mantoan H, Saito A, Salvadori MM, Guimarães AP, et al. The Value of Secondary Cytoreductive Surgery in Recurrent Ovarian Cancer and Application of a Prognostic Score. Int J Gynecol Cancer. 2016; 26(3):449-455. doi: 10.1097/ IGC.0000000000000649.

40. Takahashi A, Kato K, Matsuura M, Katsuda T, Matoda $\mathrm{M}$, Nomura $\mathrm{H}$, et al. Comparison of secondary cytoreductive surgery plus chemotherapy with chemotherapy alone for recurrent epithelial ovarian, tubal, or peritoneal carcinoma: A propensity scorematched analysis of 112 consecutive patients. Medicine (Baltimore). 2017; 96(37):e8006. doi: 10.1097/ MD.0000000000008006.

41. Felsinger M, Minar L, Weinberger V, Rovny I, Zlamal
F, Bienertova-Vasku J. Secondary cytoreductive surgery - viable treatment option in the management of platinum-sensitive recurrent ovarian cancer. Eur J Obstet Gynecol Reprod Biol. 2018; 228:154-160. doi: 10.1016/j.ejogrb.2018.06.036.

42. Bickell NA, Egorova N, Prasad-Hayes M, Franco R, Howell EA, Wisnivesky J, et al. Secondary Surgery Versus Chemotherapy for Recurrent Ovarian Cancer. Am J Clin Oncol. 2018; 41(5):458-464. doi: 10.1097/ COC.0000000000000310.

43. Gockley A, Melamed A, Cronin A, Bookman MA, Burger RA, Cristae MC, et al. Outcomes of secondary cytoreductive surgery for patients with platinumsensitive recurrent ovarian cancer. Am J Obstet Gynecol. 2019; 221(6):625.e1-625.e14. doi: 10.1016/j. ajog.2019.06.009.

44. ClinicalTrials.gov [Internet]. Surgery or chemotherapy in recurrent ovarian cancer (SOC 1 Trial)? Shanghai Gynecologic Oncology Group; 2019 [consultado el 1 de abril de 2020]. Disponible en: https://www. clinicaltrials.gov/ct2/show/NCT01611766.

Recibido 31 de agosto de 2020 Aprobado 27 de noviembre de 2020 